\title{
Maternal and fetal outcome of dengue fever during pregnancy
}

\section{Divyasree Harikumar Kanakalatha, Sreekumary Radha, Bindu Nambisan*}

Department of Obstetrics and Gynaecology, Medical College, Trivandrum, Kerala, India

Received: 07 September 2016

Accepted: 03 October 2016

\author{
*Correspondence: \\ Dr. Bindu Nambisan, \\ E-mail: bindu.nambisan1971@gmail.com
}

Copyright: () the author(s), publisher and licensee Medip Academy. This is an open-access article distributed under the terms of the Creative Commons Attribution Non-Commercial License, which permits unrestricted non-commercial use, distribution, and reproduction in any medium, provided the original work is properly cited.

\begin{abstract}
Background: With increasing rate of adult dengue fever victims, the number of infected pregnant women has also increased. Dengue, during pregnancy may be associated with various complications, including maternal mortality, preterm delivery, fetal death, low birth weight, neonatal admissions, fetal anomalies, and miscarriage. Timely intervention can improve the maternal as well as fetal outcome. The aim of the study was to assess the clinical profile, maternal and fetal outcome of dengue fever during pregnancy.

Methods: A prospective study was carried out, among the antenatal patients who were diagnosed with serologically confirmed dengue fever during a period of 18 months; at a Tertiary care Hospital in Government sector in Kerala, South India. Patients were included irrespective of period of gestation of contracting the disease and were followed up till delivery and all babies were followed up to six weeks post-partum.

Results: Thrombocytopenia $(<1.5 \mathrm{~L} / \mathrm{mm} 3)$ was found in $86.3 \%$ patients, of which $2.7 \%$ patients had Platelet count below 20,000 cells/mm3 and $4.1 \%$ patients required platelet transfusion. Other complications observed were spontaneous abortions (5.5\%); preterm birth (9.5\%), oligohydramnios $(8.2 \%)$ and antepartum hemorrhage $(4.1 \%)$. Fetal distress and meconium stained amniotic fluid were observed in $7.6 \%$ and $16.7 \%$ patients respectively. Adverse fetal outcome observed were low birth weight $(27.3 \%)$, prematurity $(23 \%)$ and $28.7 \%$ of the babies required NICU admission.

Conclusions: The gestational age at presentation of dengue fever appeared to be significant. Early onset or late onset in pregnancy appeared to have a bad prognosis. A high index of clinical suspicion is essential in any pregnant female with fever during epidemics especially in endemic areas like Kerala. Conservative medical and obstetrical management is the treatment of choice.
\end{abstract}

Keywords: Dengue fever, Dengue haemorrhagic fever, Dengue shock syndrome Thrombocytopenia

\section{INTRODUCTION}

Dengue is an acute mosquito-borne viral infection that places a significant socioeconomic and disease burden in India. ${ }^{1,2}$ Classic dengue fever (DF) is defined by the World Health Organization as an acute febrile illness with two or more of the following signs or symptoms: intense headache , retro-orbital pain, myalgia, arthralgia, rash, leukopenia and hemorrhagic manifestations.

The clinical severity of disease has a wide spectrum, and according to the World Health Organization (WHO) dengue classification scheme, there are four grades ranging from uncomplicated dengue fever (DF) to dengue hemorrhagic fever (DHF) and devastating dengue shock syndrome (DSS).

DHF is currently defined by the following four World Health Organization (WHO) criteria:

- Fever or recent history of fever lasting 2-7 days

- Any hemorrhagic manifestation.

- Thrombocytopenia (platelet count of $<100,000 / \mathrm{mm} 3$ )

- $\quad$ Evidence of increased vascular permeability 
The most common hemorrhagic manifestations are mild, and include a positive tourniquet test, skin haemorrhages (petechiae, hematomas), epistaxis (nose bleed), gingival bleeding (gum bleed), and microscopic haematuria. More serious types of hemorrhage include vaginal bleeding, hematemesis, melena, and intracranial bleeding.

Evidence of plasma leakage due to increased vascular permeability consists of at least one of the following:

- An elevated hematocrit $\geq 20 \%$ above the population means hematocrit for age and sex.

- A decline in hematocrit after volume-replacement treatment of $\geq 20 \%$ of the baseline hematocrit.

- Presence of pleural effusion or ascites detected by radiography or other imaging method.

- Hypoproteinemia or hypoalbuminemia as determined by laboratory test.

With the recent resurgences of the disease, an increasing the number of people including the pregnant women are affected. It is estimated that the risk of exposure is almost $1 \%$ during a given pregnancy in a highly endemic area like Kerala. ${ }^{3}$ Despite the high prevalence of dengue in this State, there are very few studies on the maternal and foetal consequences during pregnancy. Dengue infection in pregnancy carries the risk of hemorrhage for both the mother and the new-born. Literature search reveals an increased incidence of pre-term deliveries, low birth weight, pre-eclampsia and caesarean sections. Vertical transmission has also been noted.

The prevalence of various complications in pregnant women and its relation to gestational age has not been studied in this setting. Hence we conducted a prospective follow up study at our institution, among the antenatal women who presented with serologically proven dengue fever, to study the various maternal and fetal outcomes of dengue fever.

\section{METHODS}

Every consecutive antenatal patient diagnosed with dengue fever clinically and serologically during the study period were included in the study, irrespective of the gestational age. Antenatal patients who presented to the OPD and casualty with clinical features of dengue fever during the study period were examined and Laboratory investigations like Dengue IgM ELISA were used to confirm the diagnosis of Dengue fever.73 patients were included in this study after informed consent .

Detailed history and examination were performed with special regard to maternal age, parity, gestational age, symptoms at the time of diagnosis, platelet count and haematocrit at the time of diagnosis. Details regarding ICU admission need for transfusion and medical complications of dengue fever also were obtained and entered in the proforma.

The participants were followed up till delivery and all babies up to six weeks post-partum. Neonates of mothers who presented with dengue fever near term were monitored closely for clinical features of dengue fever and thrombocytopenia.

\section{RESULTS}

During the study period, 73 cases of maternal dengue were diagnosed a confirmed biologically.

Table 1: Distribution of subjects - gestational age at diagnosis.

\begin{tabular}{|lll|}
\hline $\begin{array}{l}\text { Gestational age at } \\
\text { diagnosis }\end{array}$ & Frequency & Percentage \\
\hline$<12$ weeks & 2 & 2.7 \\
\hline $12-20$ weeks & 13 & 17.8 \\
\hline $20-28$ weeks & 10 & 13.7 \\
\hline $28-34$ weeks & 12 & 16.4 \\
\hline $34-37$ weeks & 14 & 19.2 \\
\hline$>37$ weeks & 22 & 30.1 \\
\hline Total & 73 & 100.0 \\
\hline
\end{tabular}

Table 2: Trimester wise frequency of subjects.

\begin{tabular}{|lll|}
\hline Trimester & Frequency & Percentage \\
\hline I & 2 & 2.7 \\
\hline II & 23 & 31.5 \\
\hline III & 48 & $65.7 \%$ \\
\hline
\end{tabular}

Table 3: Variation of platelet count.

\begin{tabular}{|llllllll|}
\hline Platelet & \multicolumn{2}{c}{ Diagnosis } & \multicolumn{2}{c}{ Lowest platelet count } & \multicolumn{2}{c|}{ At delivery } \\
count & $\mathrm{N}$ & $\%$ & $\mathrm{~N}$ & $\%$ & $\mathrm{~N}$ & $\%$ \\
\hline$<20000$ & 2 & 2.7 & 5 & 6.8 & 0 & 0 \\
\hline $\begin{array}{l}21000- \\
50000\end{array}$ & 7 & 9.6 & 23 & 31.5 & 1 & 1.4 \\
\hline $\begin{array}{l}50000- \\
100000\end{array}$ & 27 & 37.0 & 19 & 26.0 & 3 & 4.4 \\
\hline $\begin{array}{l}1-1.5 \\
\text { lakh }\end{array}$ & 27 & 37.0 & 25 & 34.2 & 21 & 28.8 \\
\hline $\begin{array}{l}>1.5 \\
\text { lakhs }\end{array}$ & 10 & 13.7 & 1 & 1.4 & 43 & 58.9 \\
\hline Total & 73 & 100.0 & 73 & 100.0 & 68 & 93.1 \\
\hline
\end{tabular}

\section{Results from mothers}

\section{Epidemiology}

The cases were recruited during a period of one and half years. The mean age of the women included in our study was $26.07 \pm 4.38$ year, the youngest being $18 \mathrm{yrs}$ and the oldest being $37 \mathrm{yr}$. We had only 2 cases who had dengue fever in the first trimester of pregnancy (2/73) while $31.5 \%$ presented in the second trimester(23/73) and $65.7 \%(48 / 73)$ in the third trimester (Table 1,2$)$. 
Table 4: Fetal complications.

\begin{tabular}{|ll|}
\hline Fetal complications & Dengue \\
\hline Fetal malformations & $1(1.5)$ \\
\hline IUGR & $4(5.5)$ \\
\hline Fetal distress (intrapartum) & $5(7.6)$ \\
\hline Meconium stained amniotic fluid & $11(16.7)$ \\
\hline Still births/fetal loss & $3(4.1)$ \\
\hline
\end{tabular}

Table 5: Neonatal outcomes.

\begin{tabular}{|ll|}
\hline Neonatal outcome variables & Cases Dengue n (\%) \\
\hline Low birth weight & $21(30.3)$ \\
\hline Neonatal ICU admissions & $19(28.7)$ \\
\hline Prematurity & $14(22)$ \\
\hline $\begin{array}{l}\text { Neonatal thrombocytopenia } \\
\text { and related complications }\end{array}$ & $6(9)$ \\
\hline Vertical transmission & $2(3)$ \\
\hline
\end{tabular}

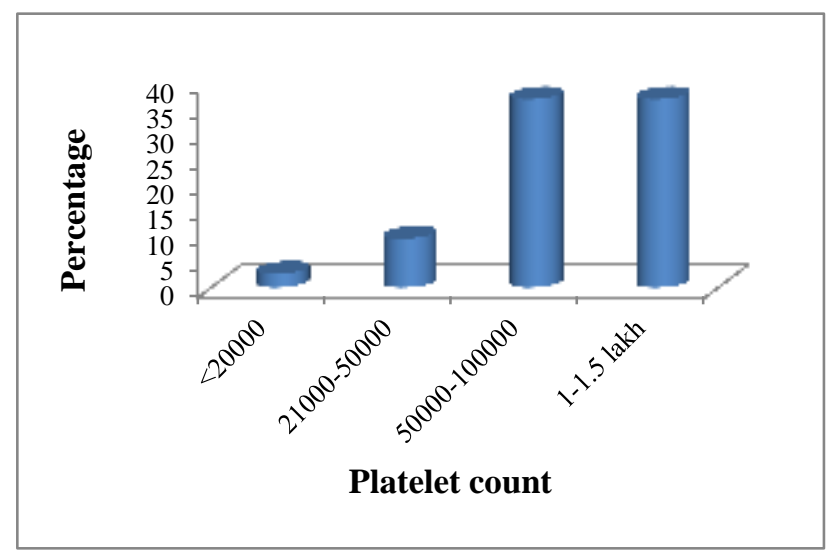

Figure 1: Platelet count at diagnosis.

\section{Clinical and biological findings}

This study had $89 \%$ patients presenting with fever, $38 \%$ with headache, $16.4 \%$ with acute abdominal pain and $21.9 \%$ with diffuse muscle pain or arthralgia as their predominant symptom. $9.6 \%$ of the patients presented with bleeding manifestations of which 3 patients $(4.1 \%)$ presented with bleeding per vaginum.

There were two cases $(2.7 \%)$ of DHF and one case (1.4\%) of DSS as described by the WHO. Of the 73 pregnant women, 5 patients $(6.8 \%)$ had an increase in liver enzymes AST (Aspartate aminotransferase) and ALT (Alanine aminotransferase) ranging from 2 to 7 times the normal level. $86.3 \%$ patients had Thrombocytopenia of which $12.3 \%$ had severe thrombocytopenia $\left(<50,000\right.$ cells $\left./ \mathrm{mm}^{3}\right)$ at the time of diagnosis, and $2.7 \%$ patients had platelet count less than 20,000 cells $/ \mathrm{mm}^{3}$ (Table 3, Figure 1).

\section{Biological diagnosis}

Of the 73 cases of maternal dengue, $95.9 \%$ of the patients were found to have Dengue IgM positive whereas $4.1 \%$ patient were found to have both Dengue $\operatorname{IgM}$ and $\operatorname{IgG}$ seropositivity.

\section{Obstetric consequences}

This study included 2 patients with dengue fever before 12 weeks of gestation of which one patient had a spontaneous abortion at 8 weeks while the other patient had an uneventful pregnancy and a full term normal delivery. Both of these patients had platelet count less than 1.5 lakhs. However, the number of patients are too less to come to any conclusion.

There were 13 patients who contracted the infection during a period of 12 to 20 weeks of gestation. Of these 2 patients had spontaneous abortion and another patient had to undergo MTP for fetal skeletal dysplasia. Among the two patients who had spontaneous abortion both of them had platelet count less than 50,000 per $\mathrm{cc}^{3}$ and required platelet transfusion. Another 4 patients had platelet counts less than $50,000 / \mathrm{cc}^{3}$ of which 2 patients developed preeclampsia later in pregnancy and required termination of pregnancy before 37 weeks. All the other patients had otherwise uncomplicated pregnancy and term delivery.

We had 10 patients who developed dengue fever during a period of 20 to 28 weeks of gestation. Of these two patients developed preterm labour, one patient developed Dengue Shock Syndrome. Four patients had platelet counts less than $1.5 \mathrm{~L}$ (of which one was DSS), one patient had preterm delivery, one had Fetal growth restriction and the other patient had uneventful pregnancy.

In our study 12 patients presented between 28 to 34 weeks of gestation, and among these patients, 5 of them (41.6\%) had preterm deliveries. One patient had Dengue haemorrhagic fever and all others had term deliveries.

Between 34 weeks and 37 weeks, 14 patients were included, of which 5 patients $(35 \%)$ had preterm deliveries.

Majority of patients (30.1\%) were infected after 37 weeks and intrapartum complications like fetal distress (13\%) and Meconium stained amniotic fluid (26\%) were more among these patients. $8.8 \%$ patients developed postpartum haemorrhage, $7.3 \%$ had postpartum fever, 7.3\% required ICU admission and $2.9 \%$ had respiratory distress in the postpartum period (Table 4).

\section{Neonatal consequences}

Birth weights were between 2500 and $3500 \mathrm{~g}$ in 31/53 cases $(65.9 \%)$. Birth weights less than $2,500 \mathrm{~g}$ were observed in $21(30.4 \%)$ new-borns, with a minimum of $1 \mathrm{~kg}$. In 3 cases, the birth weight was greater than $3500 \mathrm{~g}$; with a maximum of $3.75 \mathrm{~kg}$. Only $23 \%$ of the babies were preterm. Nineteen Babies (28.7\%) required NICU admission. Neonatal thrombocytopenia was found in $9 \%$ 
of babies. There were no cases of neonatal malformations or neonatal death (Table 5).

\section{Materno-foetal transmission}

Dengue IgM was found to be positive in $3 \%$ neonates.

\section{DISCUSSION}

The WHO criteria were strictly applied to define cases of DF. Our Study included 73 confirmed cases of Dengue fever of which 2 patients developed Dengue Haemorrhagic Fever and 1 patient had Dengue Shock Syndrome.

\section{Maternal outcomes}

One of the striking feature observed in the study by $\mathrm{S}$ Panicker et al at Coimbatore was the severe thrombocytopenia (platelet count of $<50,000 \mathrm{cell} / \mathrm{mm}^{3}$ ) which was seen in $78.5 \%$ of their women of which two women had platelet counts $<10,000$ cell $/ \mathrm{mm} 3{ }^{4}$ The fall in platelet count was rapid and progressive initially. Our study also found thrombocytopenia in 63/73 patients (86\%) of which two patients had counts less than 20,000 cells $/ \mathrm{mm}^{3}$ similar to observations in the above mentioned study.

Among the patients who presented with bleeding manifestations, two patients presented with petechiae, one patient with haemoptysis, one patient with intra cranial bleed and three patients $(4.1 \%)$ with bleeding per vaginum. All these patients had platelet counts less than 50,000 cells $/ \mathrm{mm}^{3}$. This finding is in conjunction with another study by Kulkarni et al. ${ }^{5}$ They also noticed that sixty nine percentage of the haemorrhagic manifestations occurred in patients with severe thrombocytopenia.

Nine patients (14.2\%) of dengue fever and both cases of DHF and single case of DSS required platelet transfusion. Among the 3 patients who received more than 6 pint platelet transfusion, one patient had a platelet count of 31,000 with bleeding per vaginum and another with a platelet count of 15,000.Third was a case of DHF with intracranial bleed who was referred as a case of Eclampsia at 32 weeks. Her BP was found to be high and Ultrasound abdomen showed Intra uterine fetal demise. Her blood investigations showed severe thrombocytopenia and elevated transaminases. An emergency LSCS was done after transfusing 4 pint platelet concentrate and 2 pint Fresh Frozen Plasma. Post operatively also 4 pint platelet concentrate were given. Post operatively patient continued to be stuporous and CT brain showed Sub Arachnoid Haemorrhage. Dengue NS1 was found to be positive. She was shifted to the neurology ICU and she recovered with conservative management. Tagore $\mathrm{S}$ et al has also described a similar case of a dengue haemorrhagic fever complicated by eclampsia in pregnancy. ${ }^{6}$
Most of our patients had a normal clinical presentation of acute dengue fever, one of the women had the signs and symptoms of DHF, with persistent thrombocytopenia, rising haematocrit, and fluid collection in the third space. Correction of fluid and electrolyte imbalance as well as multiple platelet transfusions was helpful and the patient recovered after treatment. Chanana $\mathrm{C}$ et al also described a similar case which was mistakenly diagnosed with haemolysis, elevated liver enzymes, and low platelet count (HELLP) syndrome. ${ }^{7}$ Cases of DHF with DIC have been reported previously by Panicker $S$ et al with patient requiring massive transfusion. ${ }^{4}$

We observed a case of Dengue Shock Syndrome in pregnancy which was successfully managed with fluid and ionotrope supports. Patient was a 24 year old primigravida referred at 26 weeks of gestation as a case of fever with hypotension and dyspnoea. Her platelet count was 1.2 lakh initially which fell to 45,000 . She had positive Dengue NS1 antigen and positive IgM. Later she developed pulmonary oedema, hemoptysis and severe hypotension. She was intubated and started on dopamine and Noradrenaline. Patient recovered with supportive management and was extubated. She had intra uterine fetal demise and expelled vaginally. Post expulsion period was uneventful. She recovered completely and was discharged.

Raised serum hepatic transaminases (AST and ALT) levels were seen in all 15 patients with DHF in the study done by Kariyawasam S et a ${ }^{8}$ in Srilanka. ${ }^{8} 100 \%$ of DHF and $60 \%$ DF patients had elevated liver enzymes in the study by Waduge et al. ${ }^{9}$ In our study five patients had elevated liver enzymes which included both DHF patients, one DSS patient and two patients with Dengue fever. Liver enzymes normalised after the acute phase of the disease. None of the patients had coagulation failure or any features of DIC.

The gestational age at presentation of dengue fever also appeared to be significant. Early onset and late onset in pregnancy appeared to have a bad prognosis. In our study, four patients had spontaneous abortion of which one patient presented at 8 weeks of gestation. Of the 13 patients who got infected between 12 and 20 weeks, three patients had spontaneous abortion. Two late miscarriages at 17 and 19 weeks of gestation were observed in another study by Carles et al also. ${ }^{10}$

There was one case of fetal anomaly which was medically terminated. She had dengue fever in the first trimester at 6 weeks of gestation and on follow up was found to have skeletal dysplasia for the baby in her routine anomaly scan and underwent Medical termination of pregnancy. Sharma et $\mathrm{al}^{11}$ reported an increased incidence of neural tube defects following dengue infection but as this defect has been demonstrated following other febrile illnesses, it may well have been due to the fever rather than to any teratogenic effect of the dengue virus per se. 
Five patients $(7.6 \%)$ developed intrapartum fetal distress and three of them required emergency LSCS. One patient had instrumental delivery and another had a normal vaginal delivery. Two of these patients were near term and went into spontaneous labour during dengue infection. Fetal distress was noted in previous studies also. Basurko $\mathrm{C}$ et al also observed fetal distress in $7.5 \%$ patients similar to our study whereas Garg $\mathrm{R}$ et al noted a higher rate $16 \%$ in their study conducted at Agra. ${ }^{12}$

There were 11 cases $(16.7 \%)$ of meconium stained amniotic fluid (MSAF). Among these, seven patients presented with dengue infection near term. Another patient with dengue infection presented with preterm labour and was done an emergency LSCS for moderate MSAF. Other two patients were infected remote from term but one of the patients had oligohydramnios and another had FGR.

Another possible effect of DF during pregnancy is bleeding due to severe thrombocytopenia in some highrisk pregnancies, such as placenta previa or retro placental hematoma. Antepartum hemorrhage was noted in 3 patients $(4.1 \%)$ of which one patient had grade II abruptio placenta. Another was a patient with placenta praevia who presented at 29 weeks of gestation with bleeding per vaginum which got controlled with supportive care and platelet transfusion for a platelet count of 30,000. She underwent elective LSCS at 38 weeks .The third patient was also a case of Type III placenta praevia who presented at 37 weeks 3 days of gestation with fever and bleeding PV with platelet count 1.2 lakhs and emergency caesarean section was done. Two pint PRC was transfused for moderate atonic PPH.

There were 6 cases $(8.8 \%)$ of PPH and all of them were medically managed. Basurko $\mathrm{C}$ et al also reported postpartum haemorrhage in $9.8 \%$ cases, one of which had DIC. However there was no case of coagulation failure or DIC in our study.

Haemorrhagic complications like post-partum haemorrhage (PPH) and abruption have been described in studies.10We also had a case of abruptio placenta in our study.

Although cases of eclampsia, pulmonary oedema, abruption and preeclampsia were noted in this study, there was no case of maternal death. Ismail et al in a recent review reported three maternal deaths out of 16 cases $(2.6 \%)$ and also noted a $50 \%$ prematurity rate. ${ }^{11}$

\section{Fetal and neonatal outcomes}

Data from other authors also showed an increase in the rate of prematurity. Carles et al in their review of 38 cases in French Guiana indicate a significant increase in prematurity and fetal death The main obstetric consequences of infection were an increase in premature births (19.6\%) and an increased risk of preterm labour $(41 \%)$ cases in the study by Basurko $\mathrm{C}$ et al. ${ }^{13,14}$

In our study 4 patients $(5.5 \%)$ had preterm labour and 4 patients $(5.5 \%)$ had preterm premature rupture of membranes (PPROM).The rate of prematurity among the babies was however $(15 \%)$.

There were three cases of Intra Uterine fetal Death in our study. Among these, one was the patient with DSS and another was the patient who presented as eclampsia. The third case of IUD observed in our study was a patient who presented with dengue fever at 38 weeks of gestation, with uterine contractions and absent fetal heart beat. She had no other co morbidities. Patient developed leaking per vaginum. Ligour was meconium stained.

The mean birth weight of babies born to mothers with dengue was $2.6 \mathrm{~kg}$. Birth weights less than 2,500 g were observed in eighteen new-borns (27.3\%), with a minimum of $1690 \mathrm{~g}$. Among these 11 babies were preterm and appeared to be having weight adequate for gestational age. Although the low birth weight in five of the term babies was most likely secondary to pregnancy induced hypertension in their mothers. The exact cause in the other two was not defined. Other studies have also showed similar outcome. ${ }^{15,16}$

\section{Fetomaternal transmission}

We observed two cases of vertical transmission rate. In both cases the maternal infection occurred around the time of delivery and they underwent emergency caesarean section for meconium stained amniotic fluid. Both babies were admitted to NICU, they developed fever with thrombocytopenia on postnatal day 3 and Dengue IgM was found to be positive. One of the baby required platelet transfusion, both babies recovered and was discharged by postnatal day 10.Similar case of vertical transmission in the neonate presenting with MSAF have been described previously as well.

\section{CONCLUSION}

Pregnancies complicated by dengue infection require close monitoring for potential maternal and fetal complications.

Dengue Haemorrhagic fever may present with features similar to Preeclampsia and HELLP. Serological tests aid in confirming the diagnosis of DHF.

The gestational age at presentation of dengue fever appeared to be significant. Early onset or late onset in pregnancy appeared to have a bad prognosis.

Effects on the fetus or new-born seem to be variable, with apparently less fetal harm occurring earlier in pregnancy. In near term disease, severe fetal or neonatal illness or 
death may occur. Such illness may also predispose the new-born to subsequent DHF.

A high index of clinical suspicion is essential in any pregnant female with fever during epidemics especially in endemic areas like Kerala.

Conservative medical and obstetrical management is the treatment of choice.

\section{Funding: No funding sources}

Conflict of interest: None declared

Ethical approval: The study was approved by the Institutional Ethics Committee

\section{REFERENCES}

1. Guzman MG, Kouri G. Dengue: an update. Lancet Infect Dis. 2002;2(1):33-42.

2. Gubler DJ. Dengue, Urbanization and Globalization: The Unholy Trinity of the 21(st) Century. Trop Med Health. 2011;39(Suppl 4):3-11.

3. Carroll ID, Toovey S, Van Gompel A. Dengue fever and pregnancy - a review and comment. Travel Med Infect Dis. 2007;5:183-8.

4. Chitra TV, Panicker S. Maternal and fetal outcome of dengue fever in pregnancy. Journal of Vector Borne Diseases. 2011;48(4):210-3.

5. Kulkarni N. Study on the effectiveness of transfusion programs in dengue patients receiving platelet transfusion. Intern Jour Transf Immunohaemat. 2012;2:11-5.

6. Tagore S, Yim C F, Kwek K. Dengue haemorrhagic fever complicated by eclampsia in pregnancy. Singapore Med J Case Report. 2007;48(10):e281.
7. Malhotra N, Chanana C, Kumar S. Dengue infection in pregnancy. Int J Gynaecol Obstet. 2006;94:131-2.

8. Kariyawasam S, Senanayake H. Dengue infections during pregnancy: case series from a tertiary care hospital in Sri Lanka. J Infect Dev Ctries. 2010;4(11):767-75.

9. Waduge R, Malavige GN, Pradeepan M, Wijeyaratne CN, Fernando S, Seneviratne SL. Dengue infections during pregnancy: a case series from Sri Lanka and review of the literature. J Clin Virol. 2006;37:27-33.

10. Carles G, Youssef M. Maternal and foetal consequences of dengue fever during pregnancy. Europ Jour Obstetrics Gynecol Reproduc Biolo. 2009;147:29-32.

11. Sharma JB, Gulati N: Potential relationship between dengue fever and neural tube defects in a northern district of India. Int J Gynaecol Obstet. 1992;39:2915.

12. Agrawal P, Garg R, Srivastava S. Pregnancy Outcome in Women with Dengue Infection in Northern India, Indian Journal of Clinical Practice. 2014;24(11).

13. Carles G, Peiffer H, Talarmin A. Effects of dengue fever during pregnancy in French Guiana. Clin Infect Dis. 1999;28:637-40.

14. Carles G, Talarmin A, Peneau C, Bertsch M. Dengue fever and pregnancy. A study of 38 cases in french Guiana. J Gynecol Obstet Biol Reprod. 2000;29:75862.

15. Adam I, Jumaa AM, Elbashir HE, Karsany MS. Maternal and perinatal outcomes of dengue in Port Sudan, Eastern Sudan. Virol J. 2010;7:153-7.

16. Sinhabahu VP, Sathananthan R. Perinatal transmission of dengue: a case report, BMC Research Notes. 2014;7:795.

Cite this article as: Kanakalatha $\mathrm{DH}$, Radha $\mathrm{S}$, Nambisan B. Maternal and fetal outcome of dengue fever during pregnancy. Int J Reprod Contracept Obstet Gynecol 2016;5:3959-64. 